\title{
Transcranial Magnetic Stimulation Studies in Alzheimer's Disease
}

\author{
Andrea Guerra, ${ }^{1}$ Federica Assenza, ${ }^{1}$ Federica Bressi, ${ }^{1,2}$ Federica Scrascia, ${ }^{1}$ \\ Marco Del Duca, ${ }^{2}$ Francesca Ursini, ${ }^{1}$ Stefano Vollaro, ${ }^{1}$ Laura Trotta, ${ }^{1}$ Mario Tombini, ${ }^{1}$ \\ Carmelo Chisari, ${ }^{3}$ and Florinda Ferreri, ${ }^{1,4}$
}

${ }^{1}$ Department of Neurology, University Campus Bio-Medico of Rome, 00128 Rome, Italy

${ }^{2}$ Department of Rehabilitation, University Campus Bio-Medico of Rome, 00128 Rome, Italy

${ }^{3}$ Department of Neuroscience, University of Pisa, 56126 Pisa, Italy

${ }^{4}$ Department of Clinical Neurophysiology, University of Eastern Finland, 70210 Kuopio, Finland

Correspondence should be addressed to Andrea Guerra, andrea.guerracbm@gmail.com and Florinda Ferreri, f.ferreri@unicampus.it

Received 30 December 2010; Revised 11 April 2011; Accepted 5 May 2011

Academic Editor: Giuseppe Curcio

Copyright (๑) 2011 Andrea Guerra et al. This is an open access article distributed under the Creative Commons Attribution License, which permits unrestricted use, distribution, and reproduction in any medium, provided the original work is properly cited.

\begin{abstract}
Although motor deficits affect patients with Alzheimer's disease (AD) only at later stages, recent studies demonstrated that primary motor cortex is precociously affected by neuronal degeneration. It is conceivable that neuronal loss is compensated by reorganization of the neural circuitries, thereby maintaining motor performances in daily living. Effectively several transcranial magnetic stimulation (TMS) studies have demonstrated that cortical excitability is enhanced in AD and primary motor cortex presents functional reorganization. Although the best hypothesis for the pathogenesis of AD remains the degeneration of cholinergic neurons in specific regions of the basal forebrain, the application of specific TMS protocols pointed out a role of other neurotransmitters. The present paper provides a perspective of the TMS techniques used to study neurophysiological aspects of AD showing also that, based on different patterns of cortical excitability, TMS may be useful in discriminating between physiological and pathological brain aging at least at the group level. Moreover repetitive TMS might become useful in the rehabilitation of AD patients. Finally integrated approaches utilizing TMS together with others neuro-physiological techniques, such as high-density EEG, and structural and functional imaging as well as biological markers are proposed as promising tool for large-scale, low-cost, and noninvasive evaluation of at-risk populations.
\end{abstract}

\section{Introduction}

Alzheimer's disease (AD) is a neurodegenerative disorder clinically characterized by a progressive cognitive decline that affects memory and other cognitive functions, as well as mood and behavior [1]. It is the most common type of dementia and nowadays it affects more than 35 million people all over the world. The disease is characterized by extracellular formation of $A \beta$ amyloid plaques and intracellular deposition of neurofibrillary tangles in specific cortical areas; this process leads to loss of neurons and white matter, amyloid angiopathy, inflammation, and oxidative damage [2]. Transcranial magnetic stimulation (TMS) is a safe, noninvasive and painless technique today widely employed to explore brain functions [3, 4]. From about 15 years, it provides a valuable tool for studying the pathophysiology of
Alzheimer's disease. This paper intends to review the most relevant studies in the literature in order to provide to the reader a clear picture of what we have learned by using TMS in the study of AD. A PubMed-based literature review of English-language studies was performed to acquire publications on AD and TMS. Key search words were "dementia, Alzheimer's disease, transcranial magnetic stimulation, and motor cortex excitability."

This work is schematically divided in to several sections: after the introduction, in Section 2 we briefly discuss basic principles of TMS and some types of paradigms used by researchers in studying AD patients; in Section 3 we explain why TMS is important for studying AD pathophysiology and what are the main $\mathrm{AD}$ alterations highlighted with this neurophysiological technique; in Section 4 we briefly introduce studies that used TMS to make differential diagnosis 
of dementia; in Section 5 we discuss TMS employment as a treatment tool and in Section 6 we provide concluding remarks and topics of future research.

\section{TMS}

Transcranial magnetic stimulation (TMS) is a safe, noninvasive, and painless technique today widely employed in studies designed to explore brain functions $[3,4]$. It was introduced by Barker and colleagues in 1985, inspired by transcranial electric stimulation studies. In TMS short current pulses are driven through a coil positioned on the scalp of the subject [6]. The transient magnetic field generated in the brain produces an electrical current able to depolarize the cell membrane, resulting in opening of voltage-gated ion channels and consequently giving rise to the action potential. The electric field induced by TMS depends on the position and orientation of the coil over the head of the subject and also by structural anatomical features and by the local conductivity of the scalp itself [7]. Different types of stimulation, declined in several type of paradigms, are currently possible (e.g., single pulse, paired-pulse, repetitive): we will focus on those more widely used in studying Alzheimer's disease.

2.1. Single Pulse. Applying a single TMS pulse over primary motor area, a series of epidurally recordable corticospinal volleys are generated which reflect the transsynaptic activation of superficial cortical neurons. Volley's temporal summation at the spinal motoneuron level elicit a motor evoked potential (MEP) in contralateral target muscles [8]. This approach is useful to study the disease processes or the neuroactive drugs [9-12] that affect regulatory mechanism of cortical excitability [13]. Single TMS pulses are also very useful to track plastic changes which originate from physiological and pathological manipulations involving the motor system and can be used for mapping motor cortical outputs. Cortical mapping procedures, with single TMS pulses focally applied on several scalp positions overlying the motor cortex, take into account the number of cortical sites eliciting MEPs in a target muscle and its "center of gravity" (COG, $[9,10])$. The location of the COG of the MEP map corresponds to the scalp location at which the largest number of the most excitable corticospinal neurons can be stimulated. Therefore, changes in the COG are considered able to indicate true changes in the topographical organization of motor cortex representations.

2.2. Paired-Pulse (SICI and ICF, SAI). TMS paired-pulse (ppTMS) protocols consist in the erogation of two individual different kinds of stimuli separated by a predetermined interval of time (interstimulus interval -ISI-). In a wellknown paradigm $[12,15]$ able to test intracortical inhibitory/facilitatory balance, a subthreshold magnetic conditioning stimulus (S1) is followed by a suprathreshold magnetic test stimulus (S2) delivered on the same target area through the same coil. The effect of S1 on the size of control MEP is thought to originate at the cortical level $[14,16,17]$. It is in fact known that a supra-threshold stimulus determines a corticospinal output leading to a MEP, while a sub-threshold stimulus only excites local, cortical interneurons [18]. Thus, by combining a sub-threshold pulse with a suprathreshold pulse one can assess the effects of inter-neurons on cortical output $[19,20]$. The test responses are inhibited at interstimulus intervals (ISIs) of $1-5 \mathrm{~ms}$ and are facilitated at ISIs of 8-30 ms; these phenomena are referred as short intracortical inhibition (SICI) and intracortical facilitation (ICF). Based on the time course of cortical inhibition and facilitation and on results of pharmacological manipulations during ppTMS, several authors have suggested that SICI is mediated by GABA-A receptors [21] whereas ICF is mediated by glutamatergic N-methyl-D-aspartate (NMDA) receptors, $[19,20]$. The balance between SICI and ICF is altered in several neurological conditions showing abnormal cortical excitability $[22,23]$.

Another paradigm, widely used in $\mathrm{AD}$, to study intracortical inhibitory mechanisms is the short-latency afferent inhibition (SAI). This approach consists of a conditioning electric stimulation applied on the median nerve at the wrist preceding a contralateral TMS test pulse by $20-25 \mathrm{~ms}$, a timing compatible to the activation of the primary sensori-motor cortex. The resulting MEPs are inhibited with respect to those evoked by the test pulse alone. The origin of this phenomenon is cortical [24-26] and probably it depends on the central cholinergic activity; Di Lazzaro and colleagues in fact clearly demonstrated that SAI can be abolished by scopolamine, a muscarinic antagonist [27].

2.3. Repetitive TMS. Repetitive TMS (rTMS) consist of single TMS pulses delivered in trains with a constant frequency and intensity for a given time. Repetitive TMS is capable to temporarily modify the function of the underlying cortical area because rTMS may exert excitatory or inhibitory actions on underling cortical activity, depending on TMS parameter used, as well as on the task at hand. An important feature of rTMS is the capacity, depending on the frequency of application, to increase or decrease the level of cortical excitability. This is the basis for the reported clinical benefits in diseases linked to brain excitability dysfunctions and the reason why this technique is increasingly used with therapeutic and rehabilitative functions $[28,29]$.

\section{TMS for Studying AD Pathophysiology}

Neurophysiological aspects usually studied in Alzheimer's disease by means of TMS are alteration of motor cortex excitability and cortical reorganization of motor output. TMS studies have in fact clearly demonstrated the existence of cortical hyperecitability and subclinical motor cortical reorganization mostly in the early stages of $\mathrm{AD}$.

The cortical hyperexcitability is believed to be a compensatory mechanism to execute voluntary movements [5], despite the progressive impairment of associative cortical areas. At present, it is not clear if these motor cortex excitability changes might be the expression of an involvement of intracortical excitatory glutamatergic circuits or an impairment of cholinergic and/or gabaergic activity [5]. In fact, although 
the best hypothesis for the pathogenesis of $\mathrm{AD}$ remains the degeneration of cholinergic neurons in specific regions of the basal forebrain, the application of specific TMS protocols, such as the paired-pulse TMS (ppTMS) and the study of the short-latency afferent inhibition (SAI; [30-32]), points out the role of other neurotransmitters, such as $\gamma$-aminobutyric acid (GABA), glutamate, and dopamine [33, 34].

\subsection{Alteration of Motor Cortex Excitability in Alzheimer's} Disease. Even though TMS evaluations in AD have not yielded absolutely converging findings, recent studies, but one [35], strongly support the hypothesis of early motor cortex global hyperexcitability in $\mathrm{AD}$ [5], opposed to the progressive hypoexcitability to TMS normally found with aging $[36,37]$. Most of these studies showed that resting motor threshold ( $\mathrm{rMT})$ is generally reduced in $\mathrm{AD}$ and in subcortical ischemic vascular dementia $(\mathrm{VaD})$ compared to healthy age-matched controls [18, 30, 32, 38-42]; however several other reports have not found reduction of motor thresholds [5, 43-45]. To date it is not yet possible to give an univocal pathophysiological interpretation of the hyperexcitability, however it could be determined mainly by two different mechanisms:

(i) increase of excitability of the intracortical excitatory circuits;

(ii) impairment of intracortical inhibitory circuits resulting in a disinhibition of the motor cortex.

As the main excitatory neurotransmitter in the brain is glutamate, the first mechanism would imply an involvement of glutamatergic transmission in AD. Indeed, several studies suggest that abnormalities of glutamatergic neurotransmission might play an important role in $\mathrm{AD}$, and the glutamatergic hypothesis of $\mathrm{AD}$ has been proposed as an auxiliary mechanism to the cholinergic hypothesis [5]; this would be due to an imbalance between non-NMDA and NMDA neurotransmission $[5,46-50]$.

However, the hypothesis of impairment of intracortical inhibitory circuits leading to disinhibition of the motor cortex in $\mathrm{AD}$ should also be taken carefully into consideration because several recent studies have demonstrated an abnormality of two inhibitory mechanisms accessible via TMS in patients with $\mathrm{AD}$, that is, SICI and SAI, respectively mediated by GABA-A receptors and cholinergic neurons activities. Liepert and colleagues in 2001 used ppTMS according to Kujirai and colleagues [15] in mildly to moderately demented $\mathrm{AD}$ patients. They found a reduced SICI compared to an age-matched control group and a correlation between the amount of disinhibition and the severity of dementia. Later, in 2004, Pierantozzi and colleagues applied the same ppTMS protocol in two groups of early-onset demented patients with a neuropsychological profile suggestive of $A D$ and frontotemporal dementia (FTD). They found a significant reduction of MEPs inhibition at ISI 2-3 ms in early-onset $\mathrm{AD}$ patients but not in controls and in FTD patients and speculated that these changes may be ascribed, at least in part, to an impaired endogenous cholinergic transmission (see also [44]) as they might be reversed by middle-term treatment with galantamine and other acetylcholinesterase inhibitors [30, 43, 44]. In 2010 also Olazarán and colleagues used the same ppTMS protocol in eleven patients with mild cognitive impairment (MCI) that converted to $\mathrm{AD}$ and 12 elderly control subjects. Cognitive assessment and ppTMS were performed at baseline in the two groups and after 4 and 21 months of treatment with donepezil in the $\mathrm{AD}$ group and ICF and SICI were found reduced in AD patients. However, there was high interindividual variability, and statistical significance was only attained at a 2 -ms interstimulus interval (ISI). A trend towards recovery of 2-ms SICI was observed after treatment with donepezil. Baseline cortical excitability at $300 \mathrm{~ms}$ was associated with better cognitive performance in $\mathrm{AD}$ patients. Anyway, although the SICI is considered to be mediated by GABA-A receptors [21], to date it is not clear if the SICI impairment observed in AD patients is really an expression of an involvement of GABAergic activity [51] as biochemical investigations of biopsy brain tissue from patients in the early phases of AD have not shown significant alterations in the concentration of GABA and no disturbance of GABA transporters $[52,53]$.

Converging evidence also suggests that SAI, an inhibitory phenomenon $[24,26,54]$ considered as a putative marker of central cholinergic activity, is reduced in $\mathrm{AD}$ and that $\mathrm{AChEI}$ (acetylcholinesterase inhibitors) therapy can rescue it $[18,32$, 55] pointing out the fact that probably the central cholinergic dysfunction occurs in early stages of Alzheimer's disease [55]. However also other neurotransmitters were recently claimed to be involved in $\mathrm{AD}$; for example, recently Martorana and colleagues [56], to test whether cholinergic disfunction could be modified by dopamine, designed a neurophysiological protocol consisting of the study of SAI before and after a single L-Dopa administration in AD patients and in healthy subjects. They observed that SAI was reduced in AD patients with respect to normal subjects, and that L-dopa administration was able to restore SAI-induced modification only in $\mathrm{AD}$. They explained these data with a relationship between acetylcholine and dopamine systems.

Finally, very recently we explored changes in cortical excitability and reorganization in AD during long-term AchEIs therapy $[14,57]$. We compared motor cortex functionality in $10 \mathrm{AD}$ patients before and after one year of AchEIs therapy and we found the examined parameters of motor cortex physiology unchanged in patients with stabilized cognitive performance during the therapy (Tables 1 and 2). Therefore, thought the study was performed in a limited number of patients, we suggested that serial TMS analysis might be a useful, non-invasive and low-cost method to monitor rate of change in motor cortex hyperexcitability in $\mathrm{AD}$, as well as AchEI CNS bioavailability and long-term pharmacological response. This idea is also supported by other experiments using the evaluation of SAI for the assessment of response to treatment $[18,31]$.

In conclusion, clinical and neuropsychological assessment are current standards to evaluate response to therapy and they are well validated, but they are somewhat dependent on examiner's expertise and, most of all, on patient's motivation. TMS could be helpful in reducing interindividual variability and achieving a more direct measure of disease progression. To date there are not univocal explanations of 


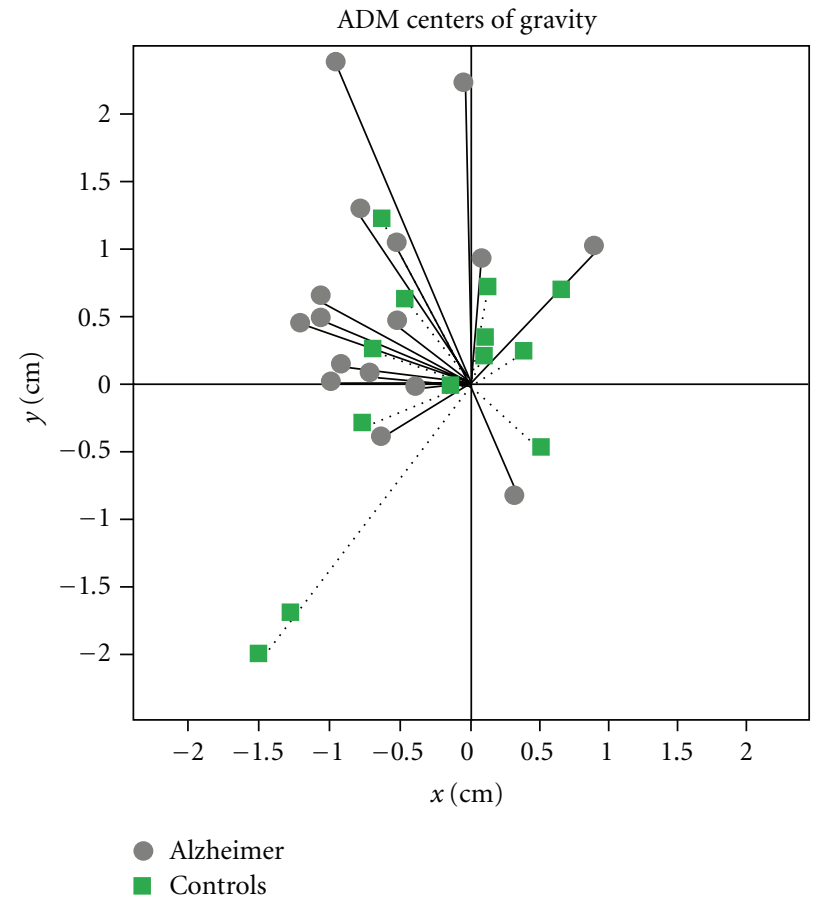

(a)

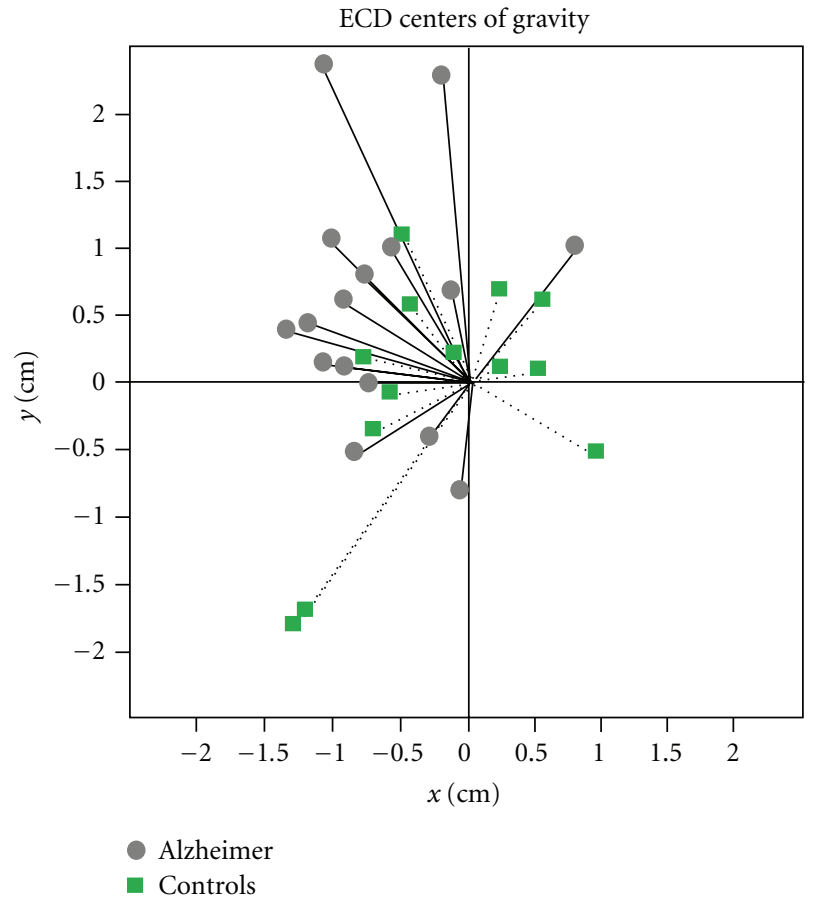

(b)

Figure 1: ADM: Abductor Digiti Minimi Muscle, ECD: Extensor Digitorum Communis Muscle. This graph shows that the map centre of gravity of the two muscles considered separately is in controls widely distributed around to hot-spot, while in patients it is evidently located anteromedial to it (modified from [5]).

TABLE 1: Mini mental state evaluation trend over two years in patients examined. $T_{1}$ : basal evaluation, $T_{2}: 1$ year after AchE-ib treatment, $T_{3}$ : 1 year after the last TMS session, DS: standard deviation. Patients, both as a group and as individual cases, could be considered cognitively stabilized at $T_{2}$ and at $T_{3}$ and formed an homogeneous group (modified from [14]).

\begin{tabular}{|c|c|c|c|c|c|c|c|}
\hline PATIENT & MMSE at $T_{1}$ & MMSE at $T_{2}$ & $\begin{array}{c}\text { Difference } \\
\text { between MMSE } \\
\text { at } T_{1} \text { and } T_{2} \\
\end{array}$ & MMSE at $T_{3}$ & $\begin{array}{c}\text { Difference } \\
\text { between MMSE } \\
\text { at } T_{1} \text { and } T_{3} \\
\end{array}$ & Total difference & \\
\hline 1 & 23 & 22 & 1 & 22 & 0 & 1 & \\
\hline 2 & 23 & 21 & 2 & 20 & 1 & 3 & \\
\hline 3 & 20 & 20 & 0 & 19 & 1 & 1 & \\
\hline 4 & 21 & 20 & 1 & 18 & 2 & 3 & \\
\hline 5 & 21 & 20 & 1 & 19 & 1 & 2 & \\
\hline 6 & 21 & 20 & 1 & 18 & 2 & 3 & \\
\hline 7 & 23 & 21 & 2 & 20 & 1 & 3 & \\
\hline 8 & 19 & 18 & 1 & 16 & 2 & 3 & \\
\hline 9 & 19 & 18 & 1 & 16 & 2 & 3 & \\
\hline \multirow[t]{3}{*}{10} & 23 & 23 & 0 & 22 & 1 & 1 & \\
\hline & & & 1 & & 1.3 & 2.18 & Media \\
\hline & & & 0.67 & & 0.67 & 0.95 & DS \\
\hline
\end{tabular}

TMS findings because the pathophysiology of AD refers to a complex involvement of different neurotransmitter systems in many brain areas. For example GABAergic dysfunctions revealed by paired-pulse TMS studies, could represent an epiphenomenon of the complex cortical excitability balance. This equilibrium is probably related to age, disease duration, and degree of cognitive impairment. In other words $\mathrm{AD}$ should be viewed as a pathological mosaic composed of numerous facets, in which the neurotransmitter question is only a piece of the problem.

3.2. Cortical Reorganization of Motor Output in AD. Motor symptoms are considered late events in the natural history of $\mathrm{AD}$ and their early occurrence makes the diagnosis less likely [5]. The delayed involvement of the motor system has been variably explained A smaller burden of neuropathological 


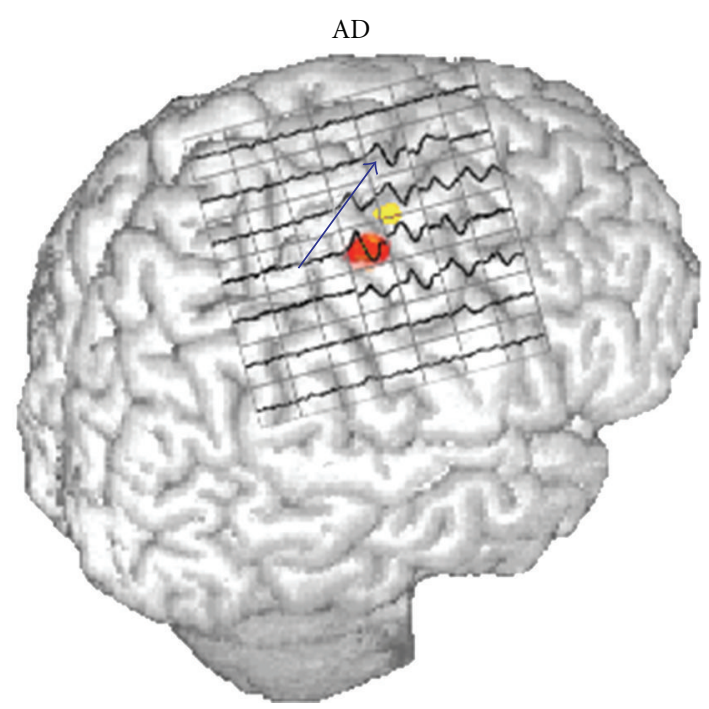

(a)

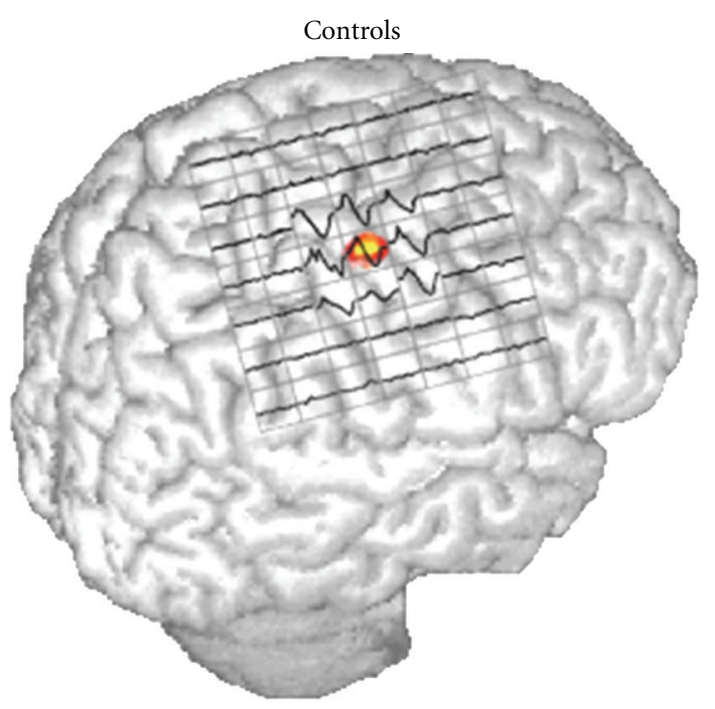

(b)

FIgURE 2: In AD patients is present a significant frontomedial shift of the center of gravity of MI output. In fact, comparing AD (a) and Controls (b) cortical maps how the hot-spot (red area) is not coincident with the center of gravity (yellow area) is evident (modified from $[5])$.

TABLE 2: Motor cortex excitability parameters trend in patients examined. $T_{1}$ : basal evaluation, $T_{2}$ : 1 year after AchE-ib treatment, ADM: Abductor Digiti Minimi Muscle, ECD: Extensor Digitorum Communis Muscle, SD: standard deviation. The table shows the AchEI therapy effect was not significantly impacting on TMS parameters (Pillai's trace $=.996 ; F(5,5)=2.440 ; P=.175$ ). Consistently, looking at single measures, the authors did not find any significant change $(P=.154$ for threshold, $P=.416$ for ADM area, $P=.484$ for ECD area, $P=.682$ for ADM volume, $P=.368$ for ECD volume) (modified from [14]).

\begin{tabular}{|c|c|c|c|c|c|}
\hline \multirow{3}{*}{ TMS Parameter } & \multirow{3}{*}{ Hemisphere } & \multicolumn{4}{|c|}{ Time } \\
\hline & & \multicolumn{2}{|c|}{$T_{1}$} & \multicolumn{2}{|c|}{$T_{2}$} \\
\hline & & Mean & SD & Mean & $\mathrm{SD}$ \\
\hline \multirow{2}{*}{ Threshold (\%) } & Right & 40.8 & 5.8 & 38.7 & 6.8 \\
\hline & Left & 39.6 & 4.9 & 37.4 & 5.6 \\
\hline \multirow{2}{*}{ Area ADM $(\mathrm{N})$} & Right & 5.3 & 2.5 & 4.9 & 2.4 \\
\hline & Left & 5.4 & 3.5 & 4.4 & 3.5 \\
\hline \multirow{2}{*}{ Area ECD $(\mathrm{N})$} & Right & 5.7 & 2.6 & 5.3 & 2.5 \\
\hline & Left & 6.1 & 4.3 & 5.2 & 3.5 \\
\hline \multirow{2}{*}{ Volume ADM (microV) } & Right & 26.8 & 12.9 & 27.0 & 15.1 \\
\hline & Left & 29.4 & 18.9 & 25.8 & 19.5 \\
\hline \multirow{2}{*}{ Volume ECD (microV) } & Right & 33.3 & 15.6 & 29.7 & 15.8 \\
\hline & Left & 38.4 & 27.6 & 31.9 & 21.0 \\
\hline
\end{tabular}

changes in the motor cortices, compared with other brain areas, a rich dendritic arborization and progressive neuronal reorganization compensatory for neural loss, have all been hypothesized. Recent neuropathological studies, though, have shown that the density of neurofibrillary tangles (NFTs) and senile plaques (SPs) in the motor cortex is comparable to that of other cortices generally considered more specific targets for $\mathrm{AD}$ pathology, such as the enthorinal cortex, the hippocampus, and the associative parietal and frontal areas [58]. Despite early modifications of motor cortex seem to be part of the neurodegenerative process in $\mathrm{AD}$ [58], the lack of early clinical manifestations might be ascribed to its ability to reorganize via alternative circuits, due to its natural distributed network with multiple representations of the motor maps [59]. The motor cortex receives a major cholinergic input from the Nucleus Basalis of Meynert, one of the most affected brain areas. TMS was employed to study the motor cortex of $\mathrm{AD}$ patients demonstrating presence of subclinical motor output reorganization from the early stages of the disease [5]. Comparison with age- and gender-matched controls showed, in the $\mathrm{AD}$ patients, increased motor cortex excitability and frontal shift of the cortical motor maps for hand and forearm muscles (Figure 1). Specifically, while in normal controls the center of gravity (CoG) of the motor cortical output, correspondent to the TMS excitable scalp sites, coincides with the site of maximal excitability, or "hot spot", [60], in AD patients the CoG showed a frontal and medial shift, with no changes in the "hot spot" location 


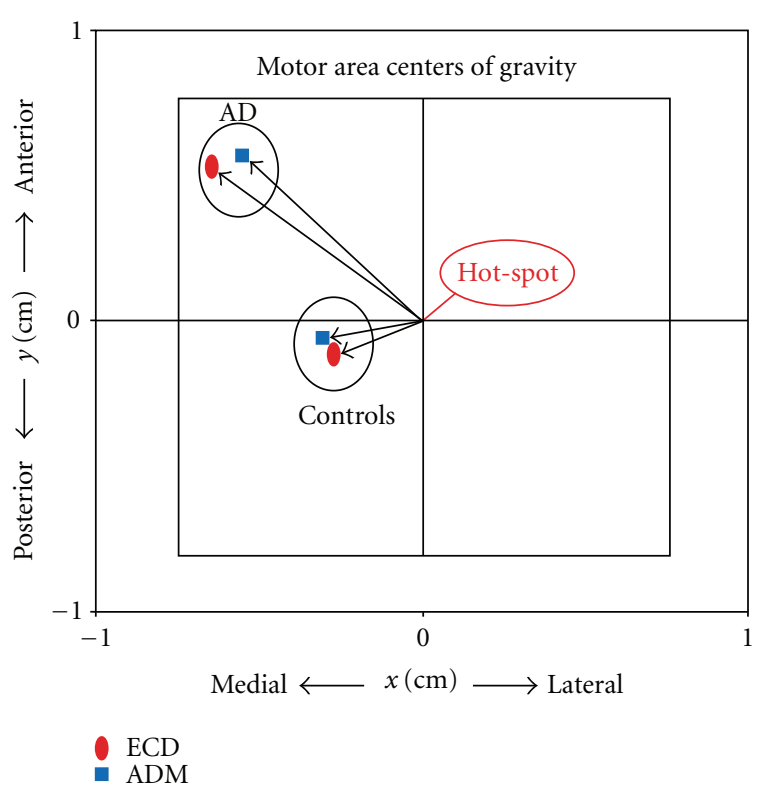

Figure 3: AD: Alzheimer disease, ADM: Abductor Digiti Minimi Muscle, ECD: Extensor Digitorum Communis Muscle, Hot-Spot: scalp site of maximal excitability. This picture shows that the coordinates of the map center of gravity compared to the hotspot appear on average significantly different in the two groups: in controls the center of gravity matches with the hot spot and is located in the center of the map. In patients there is a marked frontal and medial shift of center of gravity compared to Hot-Spot. (modified from [5]).

(Figures 2 and 3). Increased excitability and frontomedial "migration" of the excitable motor areas could be explained by neuronal reorganization, possibly including the dysregulation of the inhibitory frontal centers (the "suppressory" motor cortex or area $4 \mathrm{~S}$ ) and their integration with the distributed excitatory network subtending motor output. The frontomesial migration of the CoG does not seem to be due to gross tissue changes secondary to atrophy; were this the case, in fact, the "hot-spot" would have also similarly shifted, with no dissociation from the CoG of the map [5]. The motor cortex, for the above-mentioned reasons, seems capable of "self-defensive" reorganization, leading to late appearance of clinically evident symptoms. A more recent evidence of an altered synaptic plasticity in $\mathrm{AD}$ has been also demonstrated by Inghilleri and colleagues in 2006. They applied brief trains of high-frequency rTMS to motor cortex and recorded MEPs from the contralateral hand muscles. The researchers observed a progressive increase in MEP size in normal age-matched controls and opposite changes in $\mathrm{AD}$ [42]. This finding was interpreted as an altered short-term synaptic enhancement in excitatory circuits of the motor cortex.

\section{TMS As Potential Instrument for Differential Diagnosis of Dementia}

Recently Pierantozzi ([44], see also above) proposed the ppTMS paradigm as a noninvasive and reproducible tool to obtain an early differential diagnosis between cholinergic (AD) and non-cholinergic forms of dementia (FTD); the authors, in fact, observed a significant loss of MEPs inhibition at ISI 2-3 ms in early-onset AD patients but not in FTD patients and they speculated that these changes may be ascribed, at least in part, to an impaired endogenous cholinergic transmission.

In this vein it was also demonstrated $[32,61,62]$ that SAI is normal in FTD and in most of patients with VaD whereas it is reduced in $\mathrm{AD}$ and dementia with Lewy bodies (DLB). All together these results seem interesting with a view to find a tool to early differentiate different kind of dementia but further studies are required to confirm these data and to introduce this approach in the daily clinical practice.

\section{TMS to Improve Cognitive Performance in MCI and AD Patients}

In the last 5 years rTMS has been proposed as a possible treatment to improve cognitive performance not only in normal subjects but also in patients affected by dementia in which it may represent a useful tool for cognitive rehabilitation. Particularly on one hand it was demonstrated that rTMS induce a transient improvement in the associative memory task in normal subjects and that it is associated with recruitment of right prefrontal and bilateral posterior cortical regions [63], on the other hand further studies have demonstrated that rTMS on dorsolateral prefrontal cortex improves naming performance in mild AD patients and also in the advanced stages of the disease $[64,65]$. Moreover recently rTMS [66] was applied to AD patients to assess the duration of its effects on language performance and it was found that a 4 -week daily real rTMS treatment is able to induce at least an 8-week lasting effect on the improved performance.

Bentwich and colleagues [67] combined rTMS (applied on six different brain regions) with cognitive training; they recruited eight patients with probable $\mathrm{AD}$, who were treated for more than 2 months with cholinesterase inhibitors. These patients were subjected to daily rTMS-cognitive training sessions (5/week) for 6 weeks, followed by maintenance session (2/week) for an additional 3 months. They demonstrated a significant improvement in Alzheimer Disease Assessment Scale-Cognitive (ADAS-cog) and in Clinical Global Impression of Change (CGIC) after both 6 weeks and 4.5 months of treatment. These findings represent direct evidences that rTMS is helpful in restoring brain functions and could reflect rTMS potential to recruit compensatory networks that underlie the memory-encoding and other congnitive processes [68].

\section{Conclusions and Future Perspectives}

Initially developed to excite peripheral nerves, TMS was quickly recognized as a valuable tool to noninvasively investigate and even activate the cerebral cortex in several neuro-psychiatric disorders, such as dementia; to date the all findings available suggest that TMS is a valuable tool to study the neurophysiological basis of cognitive disorders. TMS 
furnished several interesting patho-physiological information and, although the cholinergic deficit seems to be the most accepted hypothesis, recent results indicate that $\mathrm{AD}$ should be considered as a complex neurodegenerative disease, involving different neurotransmitter systems. The subsequent discovery that repetitive TMS could have long-lasting effects on cortical excitability spawned a broad interest in the use of this technique and, despite the current outcomes from initial trials include some conflicting results, initial evidence supports the idea that rTMS might have some therapeutic value in $\mathrm{AD}$. To date, few studies have been conducted at the predementia stage and correlations between cortical excitability and cognitive performance have not been clearly addressed.

Finally as altered functional connectivity may precede structural changes, an objective method for the investigation of early functional changes in cortical connectivity might be useful in the early diagnosis and followup of $\mathrm{AD}$ [69]. With this view the combined use of TMS with other brain mapping techniques will greatly expand the scientific potential of TMS in basic neuroscience and clinical research and will provide substantial new insights in the pathophysiology of neuropsychiatric diseases. In recent years, several commercially available devices have been introduced that allow recording electroencephalographic (EEG) responses to TMS of a given scalp site with millisecond resolution. The latency, amplitude, and scalp topography of such responses are considered a reliably reflection of corticocortical connectivity and functional state $[14,57,70]$. Combining TMS with EEG enables a noninvasive, finally direct, method to study cortical excitability and connectivity that is intrinsic neural properties and the connections of thalamocortical circuits can be explored without involve peripheral stimulation or requiring the active engagement of subjects in a cognitive task. EEGTMS is a promising tool to better characterize the neuronal circuits underlying cortical effective connectivity and its disruption in Alzheimer's disease and other kind of dementia [69].

\section{Acknowledgments}

Preparation of this paper was partially supported by the Grant GR-2008-1143091 from the Italian Institute of Health. The authors gratefully thanks Professor Paolo Maria Rossini for his continuous support.

\section{References}

[1] S. Borson and M. A. Raskind, "Clinical features and pharmacological treatment of behavioural symptoms of Alzheimer's disease," Neurology, vol. 48, no. 5, supplement 6, pp. S17-S24, 1997.

[2] H. W. Querfurth and F. M. LaFerla, "Alzheimer's disease," New England Journal of Medicine, vol. 362, no. 4, pp. 329-344, 2010.

[3] M. Kobayashi and A. Pascual-Leone, "Transcranial magnetic stimulation in neurology," Lancet Neurology, vol. 2, no. 3, pp. 145-156, 2003.

[4] P. M. Rossini and S. Rossi, "Transcranial magnetic stimulation: diagnostic, therapeutic, and research potential," Neurology, vol. 68, no. 7, pp. 484-488, 2007.
[5] F. Ferreri, F. Pauri, P. Pasqualetti, R. Fini, G. Dal Forno, and P. M. Rossini, "Motor cortex excitability in Alzheimer's disease: a transcranial magnetic stimulation study," Annals of Neurology, vol. 53, no. 1, pp. 102-108, 2003.

[6] A. T. Barker, R. Jalinous, and I. L. Freeston, "Non-invasive magnetic stimulation of human motor cortex," Lancet, vol. 1, no. 8437, pp. 1106-1107, 1985.

[7] P. T. Fox, S. Narayana, N. Tandon et al., "Column-based model of electric field excitation of cerebral cortex," Human Brain Mapping, vol. 22, no. 1, pp. 1-14, 2004.

[8] V. Di Lazzaro, A. Oliviero, P. Profice et al., "Descending spinal cord volleys evoked by transcranial magnetic and electrical stimulation of the motor cortex leg area in conscious humans," Journal of Physiology, vol. 537, no. 3, pp. 1047-1058, 2001.

[9] P. M. Rossini, G. Martino, L. Narici et al., "Short-term brain "plasticity" in humans: transient finger representation changes in sensory cortex somatotopy following ischemic anesthesia," Brain Research, vol. 642, no. 1-2, pp. 169-177, 1994.

[10] P. M. Rossini, A. T. Barker, A. Berardelli et al., "Noninvasive electrical and magnetic stimulation of the brain, spinal cord and roots: basic principles and procedures for routine clinical application. Report of an IFCN committee," Electroencephalography and Clinical Neurophysiology, vol. 91, no. 2, pp. 79-92, 1994.

[11] P. M. Rossini, A. Berardelli, G. Deuschl et al., "Applications of magnetic cortical stimulation. The International Federation of Clinical Neurophysiology," Electroencephalography and Clinical Neurophysiology, vol. 52, supplement, pp. 171-185, 1999.

[12] U. Ziemann, "TMS and drugs," Clinical Neurophysiology, vol. 115, no. 8, pp. 1717-1729, 2004.

[13] P. M. Rossini, S. Rossi, C. Babiloni, and J. Polich, "Clinical neurophysiology of aging brain: from normal aging to neurodegeneration," Progress in Neurobiology, vol. 83, no. 6, pp. 375-400, 2007.

[14] F. Ferreri, P. Pasqualetti, S. Määttä et al., "Motor cortex excitability in Alzheimer's disease: a transcranial magnetic stimulation follow-up study," Neuroscience Letters, vol. 492, no. 2, pp. 94-98, 2011.

[15] T. Kujirai, M. D. Caramia, J. C. Rothwell et al., "Corticocortical inhibition in human motor cortex," Journal of Physiology, vol. 471, pp. 501-519, 1993.

[16] T. Shimizu, M. Oliveri, M. M. Filippi, M. G. Palmieri, P. Pasqualetti, and P. M. Rossini, "Effect of paired transcranial magnetic stimulation on the cortical silent period," Brain Research, vol. 834, no. 1-2, pp. 74-82, 1999.

[17] M. Orth, A. H. Snijders, and J. C. Rothwell, "The variability of intracortical inhibition and facilitation," Clinical Neurophysiology, vol. 114, no. 12, pp. 2362-2369, 2003.

[18] V. Di Lazzaro, A. Oliviero, P. A. Tonali et al., "Noninvasive in vivo assessment of cholinergic cortical circuits in $\mathrm{AD}$ using transcranial magnetic stimulation," Neurology, vol. 59, no. 3, pp. 392-397, 2002.

[19] U. Ziemann, F. Tergau, E. M. Wassermann, S. Wischer, J. Hildebrandt, and W. Paulus, "Demonstration of facilitatory I wave interaction in the human motor cortex by paired transcranial magnetic stimulation," Journal of Physiology, vol. 511, no. 1, pp. 181-190, 1998.

[20] U. Ziemann, M. Hallett, and L. G. Cohen, "Mechanisms of deafferentation-induced plasticity in human motor cortex," Journal of Neuroscience, vol. 18, no. 17, pp. 7000-7007, 1998.

[21] R. Hanajima, Y. Ugawa, Y. Terao et al., "Paired-pulse magnetic stimulation of the human motor cortex: differences among I waves," Journal of Physiology, vol. 509, no. 2, pp. 607-618, 1998. 
[22] T. D. Sanger, R. R. Garg, and R. Chen, "Interactions between two different inhibitory systems in the human motor cortex," Journal of Physiology, vol. 530, no. 2, pp. 307-317, 2001.

[23] F. Ferreri, G. Curcio, P. Pasqualetti, L. De Gennaro, R. Fini, and P. M. Rossini, "Mobile phone emissions and human brain excitability," Annals of Neurology, vol. 60, no. 2, pp. 188-196, 2006.

[24] R. Mariorenzi, F. Zarola, M. D. Caramia, C. Paradiso, and P. M. Rossini, "Non-invasive evaluation of central motor tract excitability changes following peripheral nerve stimulation in healthy humans," Electroencephalography and Clinical Neurophysiology, vol. 81, no. 2, pp. 90-101, 1991.

[25] K. Stefan, E. Kunesch, L. G. Cohen, R. Benecke, and J. Classen, "Induction of plasticity in the human motor cortex by paired associative stimulation," Brain, vol. 123 , no. 3, pp. 572-584, 2000.

[26] H. Tokimura, V. Di Lazzaro, Y. Tokimura et al., "Short latency inhibition of human hand motor cortex by somatosensory input from the hand," Journal of Physiology, vol. 523, no. 2, pp. 503-513, 2000.

[27] V. Di Lazzaro, A. Oliviero, P. Profice et al., "Muscarinic receptor blockade has differential effects on the excitability of intracortical circuits in the human motor cortex," Experimental Brain Research, vol. 135, no. 4, pp. 455-461, 2000.

[28] A. Post and M. E. Keck, "Transcranial magnetic stimulation as a therapeutic tool in psychiatry: what do we know about the neurobiological mechanisms?" Journal of Psychiatric Research, vol. 35, no. 4, pp. 193-215, 2001.

[29] R. E. Hoffman and I. Cavus, "Slow transcranial magnetic stimulation, long-term depotentiation, and brain hyperexcitability disorders," American Journal of Psychiatry, vol. 159, no. 7, pp. 1093-1102, 2002.

[30] V. Di Lazzaro, A. Oliviero, F. Pilato et al., "Motor cortex hyperexcitability to transcranial magnetic stimulation in Alzheimer's disease," Journal of Neurology, Neurosurgery and Psychiatry, vol. 75, no. 4, pp. 555-559, 2004.

[31] V. Di Lazzaro, A. Oliviero, F. Pilato et al., "Neurophysiological predictors of long term response to AChE inhibitors in $\mathrm{AD}$ patients," Journal of Neurology, Neurosurgery and Psychiatry, vol. 76, no. 8, pp. 1064-1069, 2005.

[32] V. Di Lazzaro, F. Pilato, M. Dileone et al., "In vivo cholinergic circuit evaluation in frontotemporal and Alzheimer dementias," Neurology, vol. 66, no. 7, pp. 1111-1113, 2006.

[33] G. Pennisi, R. Ferri, M. Cantone et al., "A review of transcranial magnetic stimulation in vascular dementia," Dementia and Geriatric Cognitive Disorders, vol. 31, no. 1, pp. 71-80, 2011.

[34] G. Pennisi, R. Ferri, G. Lanza et al., "Transcranial magnetic stimulation in Alzheimer's disease: a neurophysiological marker of cortical hyperexcitability," Journal of Neural Transmission, vol. 118, no. 4, pp. 587-598, 2011.

[35] A. Perretti, D. Grossi, N. Fragassi et al., "Evaluation of the motor cortex by magnetic stimulation in patients with Alzheimer disease," Journal of the Neurological Sciences, vol. 135, no. 1, pp. 31-37, 1996.

[36] P. M. Rossini, M. T. Desiato, and M. D. Caramia, "Age-related changes of motor evoked potentials in healthy humans: noninvasive evaluation of central and peripheral motor tracts excitability and conductivity," Brain Research, vol. 593, no. 1, pp. 14-19, 1992.

[37] A. Peinemann, C. Lehner, B. Conrad, and H. R. Siebner, "Agerelated decrease in paired-pulse intracortical inhibition in the human primary motor cortex," Neuroscience Letters, vol. 313, no. 1-2, pp. 33-36, 2001.
[38] M. De Carvalho, A. De Mendonça, P. C. Miranda, C. Garcia, and M. De Lourdes Sales Luís, "Magnetic stimulation in Alzheimer's disease," Journal of Neurology, vol. 244, no. 5, pp. 304-307, 1997.

[39] J. L. Pepin, D. Bogacz, V. De Pasqua, and P. J. Delwaide, "Motor cortex inhibition is not impaired in patients with Alzheimer's disease: evidence from paired transcranial magnetic stimulation," Journal of the Neurological Sciences, vol. 170, no. 2, pp. 119-123, 1999.

[40] G. Alagona, R. Bella, R. Ferri et al., "Transcranial magnetic stimulation in Alzheimer disease: motor cortex excitability and cognitive severity," Neuroscience Letters, vol. 314, no. 1-2, pp. 57-60, 2001.

[41] G. Pennisi, G. Alagona, R. Ferri et al., "Motor cortex excitability in Alzheimer disease: one year follow-up study," Neuroscience Letters, vol. 329, no. 3, pp. 293-296, 2002.

[42] M. Inghilleri, A. Conte, V. Frasca et al., "Altered response to rTMS in patients with Alzheimer's disease," Clinical Neurophysiology, vol. 117, no. 1, pp. 103-109, 2006.

[43] J. Liepert, K. J. Bär, U. Meske, and C. Weiller, "Motor cortex disinhibition in Alzheimer's disease," Clinical Neurophysiology, vol. 112, no. 8, pp. 1436-1441, 2001.

[44] M. Pierantozzi, M. Panella, M. G. Palmieri et al., "Different TMS patterns of intracortical inhibition in early onset Alzheimer dementia and frontotemporal dementia," Clinical Neurophysiology, vol. 115, no. 10, pp. 2410-2418, 2004.

[45] R. Nardone, A. Bratti, and F. Tezzon, "Motor cortex inhibitory circuits in dementia with Lewy bodies and in Alzheimer's disease," Journal of Neural Transmission, vol. 113, no. 11, pp. 1679-1684, 2006.

[46] V. Di Lazzaro, A. Oliviero, F. Pilato et al., "Motor cortex hyperexcitability to transcranial magnetic stimulation in Alzheimer's disease: evidence of impaired glutamatergic neurotransmission?" Annals of Neurology, vol. 53, no. 6, pp. 824-825, 2003.

[47] V. Di Lazzaro, A. Oliviero, P. Profice et al., "Ketamine increases human motor cortex excitability to transcranial magnetic stimulation," Journal of Physiology, vol. 547, no. 2, pp. 485496, 2003.

[48] M. R. Farlow, "NMDA receptor antagonists: a new therapeutic approach for Alzheimer's disease," Geriatrics, vol. 59, no. 6, pp. 22-27, 2004.

[49] M. R. Hynd, H. L. Scott, and P. R. Dodd, "Glutamate-mediated excitotoxicity and neurodegeneration in Alzheimer's disease," Neurochemistry International, vol. 45, no. 5, pp. 583-595, 2004.

[50] T. Voisin, E. Reynish, F. Portet, H. Feldman, and B. Vellas, "What are the treatment options for patients with severe Alzheimer's disease?” CNS Drugs, vol. 18, no. 9, pp. 575-583, 2004.

[51] P. T. Francis, A. M. Palmer, M. Snape, and G. K. Wilcock, "The cholinergic hypothesis of Alzheimer's disease: a review of progress," Journal of Neurology Neurosurgery and Psychiatry, vol. 66, no. 2, pp. 137-147, 1999.

[52] S. L. Lowe, D. M. Bowen, P. T. Francis, and D. Neary, "Ante mortem cerebral amino acid concentrations indicate selective degeneration of glutamate-enriched neurons in Alzheimer's disease," Neuroscience, vol. 38, no. 3, pp. 571-577, 1990.

[53] S. L. Lowe, P. T. Francis, A. W. Procter, A. M. Palmer, A. N. Davison, and D. M. Bowen, "Gamma-aminobutyric acid concentration in brain tissue at two stages of Alzheimer's disease," Brain, vol. 111, no. 4, pp. 785-799, 1988.

[54] K. Stefan, E. Kunesch, L. G. Cohen, R. Benecke, and J. Classen, "Induction of plasticity in the human motor cortex by paired associative stimulation," Brain, vol. 123, no. 3, pp. 572-584, 2000. 
[55] R. Nardone, J. Bergmann, M. Kronbichler et al., "Abnormal short latency afferent inhibition in early Alzheimer's disease: a transcranial magnetic demonstration," Journal of Neural Transmission, vol. 115, no. 11, pp. 1557-1562, 2008.

[56] A. Martorana, F. Mori, Z. Esposito et al., "Dopamine modulates cholinergic cortical excitability in Alzheimer's disease patients," Neuropsychopharmacology, vol. 34, no. 10, pp. 2323$2328,2009$.

[57] F. Ferreri, P. Pasqualetti, S. Määttä et al., "Human brain connectivity during single and paired pulse transcranial magnetic stimulation," NeuroImage, vol. 54, no. 1, pp. 90-102, 2011.

[58] D. Suvà, I. Favre, R. Kraftsik, M. Esteban, A. Lobrinus, and J. Miklossy, "Primary motor cortex involvement in Alzheimer disease," Journal of Neuropathology and Experimental Neurology, vol. 58, no. 11, pp. 1125-1134, 1999.

[59] J. N. Sanes and J. P. Donoghue, "Plasticity and primary motor cortex," Annual Review of Neuroscience, vol. 23, pp. 393-415, 2000.

[60] P. Cicinelli, R. Traversa, A. Bassi, G. Scivoletto, and P. M. Rossini, "Interhemispheric differences of hand muscle representation in human motor cortex," Muscle and Nerve, vol. 20, no. 5, pp. 535-542, 1997.

[61] V. Di Lazzaro, F. Pilato, M. Dileone et al., "Functional evaluation of cerebral cortex in dementia with Lewy bodies," NeuroImage, vol. 37, no. 2, pp. 422-429, 2007.

[62] V. Di Lazzaro, F. Pilato, M. Dileone et al., "In vivo functional evaluation of central cholinergic circuits in vascular dementia," Clinical Neurophysiology, vol. 119, no. 11, pp. 2494-2500, 2008.

[63] C. Solé-Padullés, D. Bartrés-Faz, C. Junqué et al., "Repetitive transcranial magnetic stimulation effects on brain function and cognition among elders with memory dysfunction. A randomized sham-controlled study," Cerebral Cortex, vol. 16, no. 10, pp. 1487-1493, 2006.

[64] M. Cotelli, R. Manenti, S. F. Cappa et al., "Effect of transcranial magnetic stimulation on action naming in patients with Alzheimer disease," Archives of Neurology, vol. 63, no. 11, pp. 1602-1604, 2006.

[65] M. Cotelli, R. Manenti, S. F. Cappa, O. Zanetti, and C. Miniussi, "Transcranial magnetic stimulation improves naming in Alzheimer disease patients at different stages of cognitive decline," European Journal of Neurology, vol. 15, no. 12, pp. 1286-1292, 2008.

[66] M. Cotelli, M. Calabria, R. Manenti et al., "Improved language performance in Alzheimer disease following brain stimulation," Journal of Neurology, Neurosurgery and Psychiatry, vol. 82, no. 7, pp. 794-797, 2011.

[67] J. Bentwich, E. Dobronevsky, S. Aichenbaum et al., "Beneficial effect of repetitive transcranial magnetic stimulation combined with cognitive training for the treatment of Alzheimer's disease: a proof of concept study," Journal of Neural Transmission, vol. 118, no. 3, pp. 463-471, 2011.

[68] S. Rossi and P. M. Rossini, "TMS in cognitive plasticity and the potential for rehabilitation," Trends in Cognitive Sciences, vol. 8, no. 6, pp. 273-279, 2004.

[69] P. Julkunen, A. M. Jauhiainen, S. Westerén-Punnonen et al., "Navigated TMS combined with EEG in mild cognitive impairment and Alzheimer's disease: a pilot study," Journal of Neuroscience Methods, vol. 172, no. 2, pp. 270-276, 2008.

[70] R. J. Ilmoniemi, J. Virtanen, J. Ruohonen et al., "Neuronal responses to magnetic stimulation reveal cortical reactivity and connectivity," Neuroreport, vol. 8, no. 16, pp. 3537-3540, 1997. 


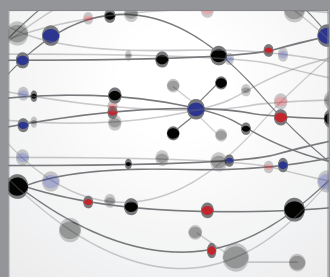

The Scientific World Journal
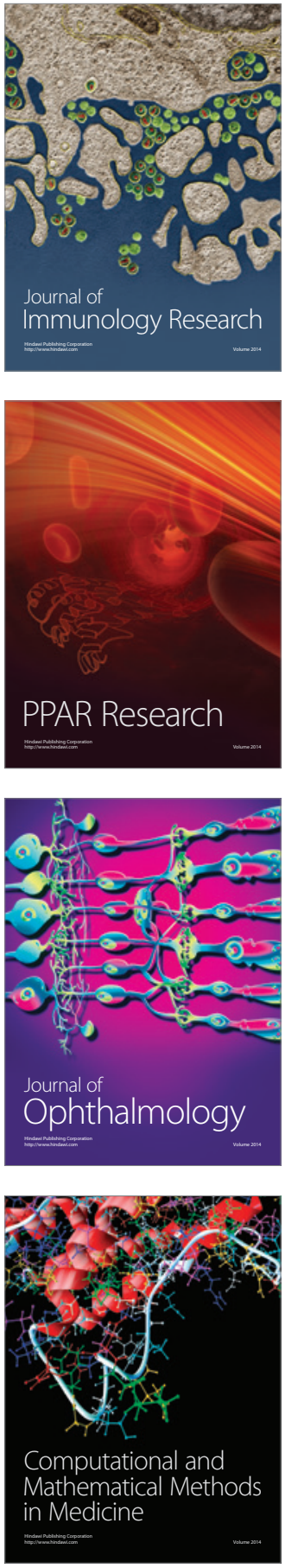

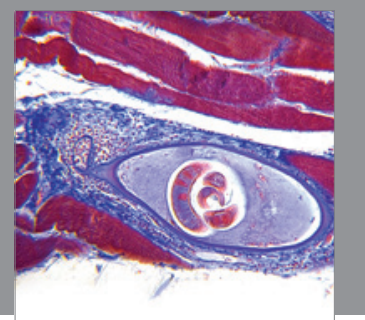

Gastroenterology

Research and Practice
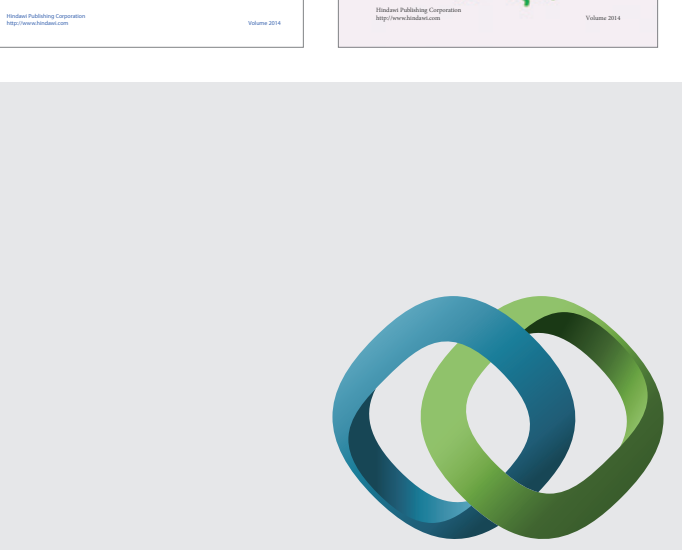

\section{Hindawi}

Submit your manuscripts at

http://www.hindawi.com
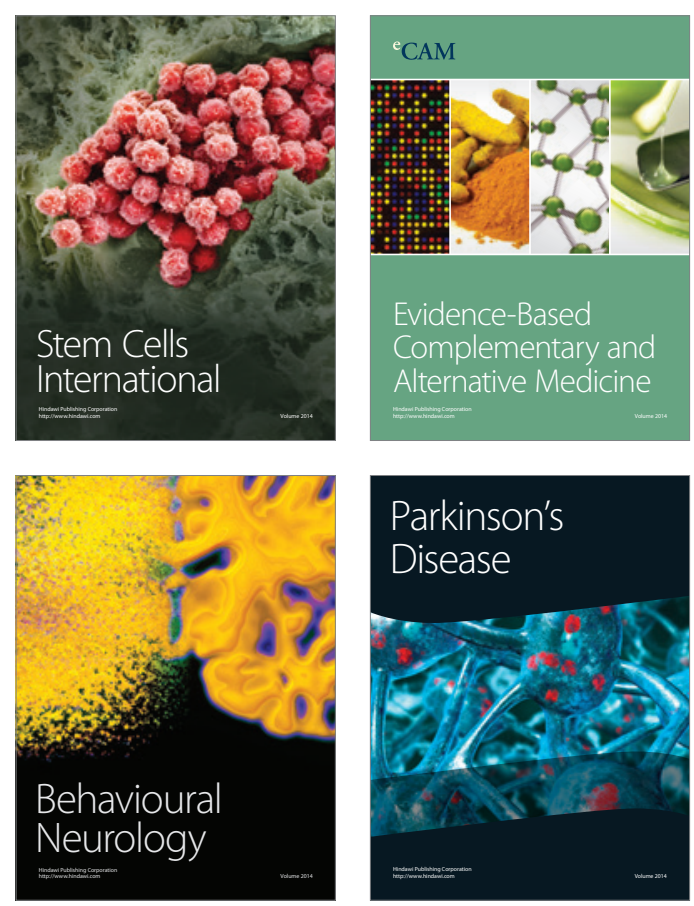

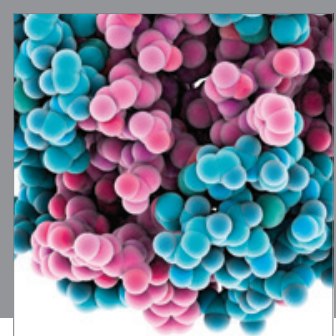

Journal of
Diabetes Research

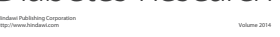

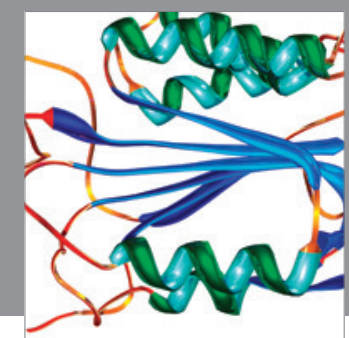

Disease Markers
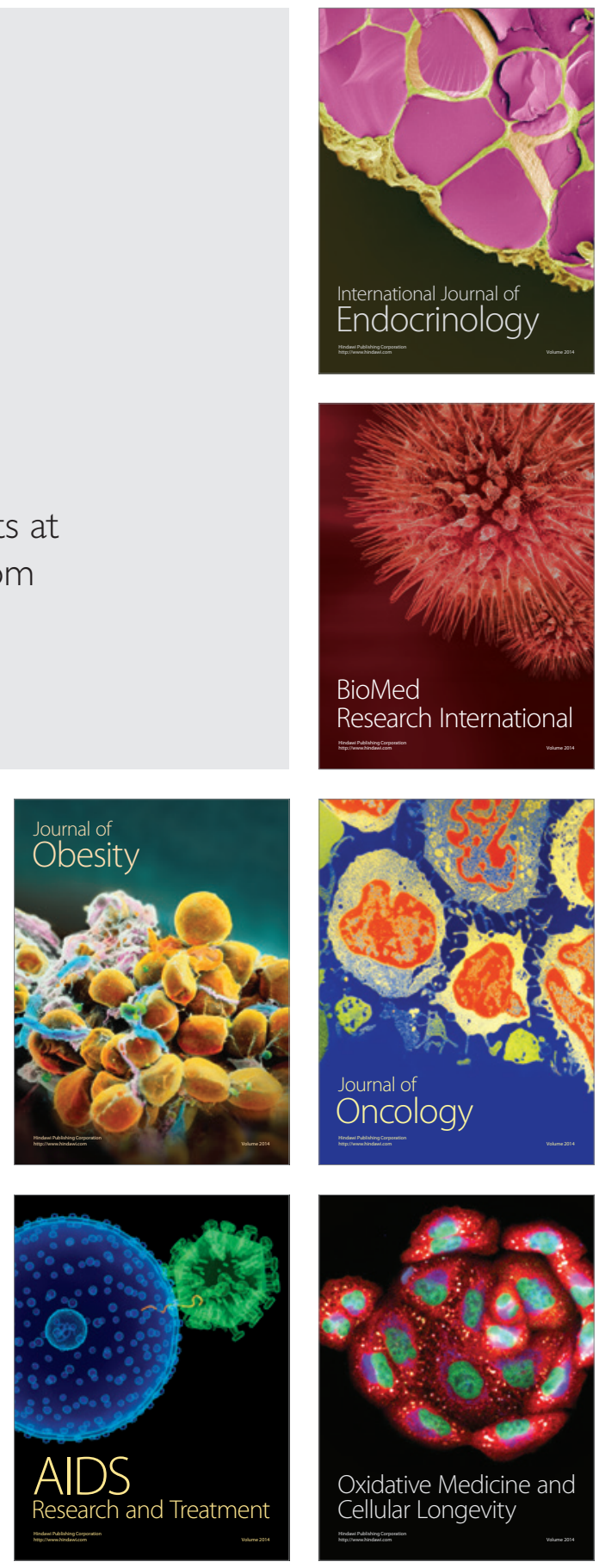